\section{Eritoran insight for influenza treatment}

\section{By Tracey Baas, Senior Editor}

A University of Maryland School of Medicine team has used a toll-like receptor 4 antagonist to treat influenza in mice. ${ }^{1}$ The results provide a repurposing opportunity for Eisai Co. Ltd.'s toll-like receptor 4 blocker Eritoran, originally developed for sepsis, and could extend to treating respiratory infections beyond the flu.

Vaccines and antivirals - namely neuraminidase inhibitors-provide the standard of care for influenza. But the limited efficacy of vaccines together with the increasing resistance to antivirals and their short therapeutic window has spurred a need for alternative strategies.

Previous work in mice has shown that infection with influenza virus, SARS or anthrax induces the production of cellular oxidized phospholipids and other molecules that activate toll-like receptor 4 (TLR4) signaling. ${ }^{2}$ This activation, which is independent of the receptor's usual ligand, lipopolysaccharide (LPS), triggers a cytokine storm that can result in acute lung injury.

Stefanie Vogel, a professor of microbiology and immunology at the University of Maryland School of Medicine, showed in 2010 that Tlr4deficient mice could survive lethal challenge with mouse-adapted H1N1 influenza A virus, whereas wild-type mice could not. ${ }^{3}$
Based on those data, Vogel and her team hypothesized that pharmacological blockade of TLR4 might provide protection against influenza and other respiratory infections. To test this, the researchers turned to Eisai's Eritoran (E5564), a synthetic lipid A analog that failed to reduce mortality in a Phase III sepsis trial in 2011. ${ }^{4}$

The team included researchers from Sigmovir Biosystems Inc., the Cincinnati Children's Research Foundation, The University of Iowa, the Iowa City VA Health Care System and Eisai's subsidiary Eisai Inc. and was co-led by Kari Ann Shirey, assistant professor of microbiology and immunology at the University of Maryland School of Medicine.

Vogel's team lethally challenged mice with a mouse-adapted H1N1 influenza A virus or a nonadapted $2009 \mathrm{H} 1 \mathrm{~N} 1$ human pandemic influenza. In both cases, daily injection of Eritoran decreased clinical symptoms, lung pathology and cytokine signaling and increased survival compared with vehicle injection, even when started as late as six days postinfection. Eritoran also decreased lung pathology compared with vehicle in human $\mathrm{H} 3 \mathrm{~N} 2$ influenza A-infected cotton rats.

In cell culture, Eritoran did not decrease levels of virus replication, indicating that it did not directly target the virus. Instead, the team hypothesized that the compound worked by blunting the cytokine storm following infection.

Indeed, mice infected with influenza and treated with Eritoran had substantially lower levels of oxidized phospholipids in their lungs than untreated mice. Eritoran also decreased pulmonary expression of many proinflammatory and anti-inflammatory cytokines, as well as interferon- $\beta$ (IFNB; IFN- $\beta$ ).

Next, the researchers looked at the effects of Eritoran on TLR4 activation. Activation of TLR4 is triggered by pathogen-induced, hostoxidized phospholipids that first bind to CD14 and then are transferred to lymphocyte antigen 96 (LY96; MD2). The lipid-MD2 complex interacts with TLR4, resulting in receptor dimerization and activation.
Figure 1. Proposed model of Eritoran-mediated protection in influenza infection. Oxidized phospholipids, produced in response to viral infection, are proposed to bind to CD14 before being transferred to lymphocyte antigen 96 (LY96; MD2). The lipid-MD2 complex interacts with toll-like receptor 4 (TLR4), resulting in receptor dimerization and activation.

Intracellular adaptor proteins - toll-interleukin 1 receptor domain containing adaptor protein (TIRAP), toll-like receptor adaptor molecule 2 (TICAM2; TRAM), myeloid differentiation primary response gene 88 (MYD88) and TICAM1 (TRIF) - are then recruited, and the signal is transmitted inside the cell. TLR4 signaling that occurs through MYD88 activates NF- $\mathrm{BB}$ to induce production of proinflammatory cytokines, whereas TLR4 signaling that occurs through TRIF activates interferon regulatory factor 3 (IRF3) to induce production of type I interferons.

Points in the signaling pathway at which Eritoran may block influenza virus-induced inflammation are indicated in red.

Vogel's group speculates that Eritoran can inhibit interactions of oxidized phospholipids with either CD14 or MD2, thus diminishing TLR4 signaling and production of cytokines. This would blunt the effect of cytokines on reactive oxygen species (ROS) generation and therefore limit the production of oxidized phospholipids. (Figure based on Figure 8 in ref. 1.)

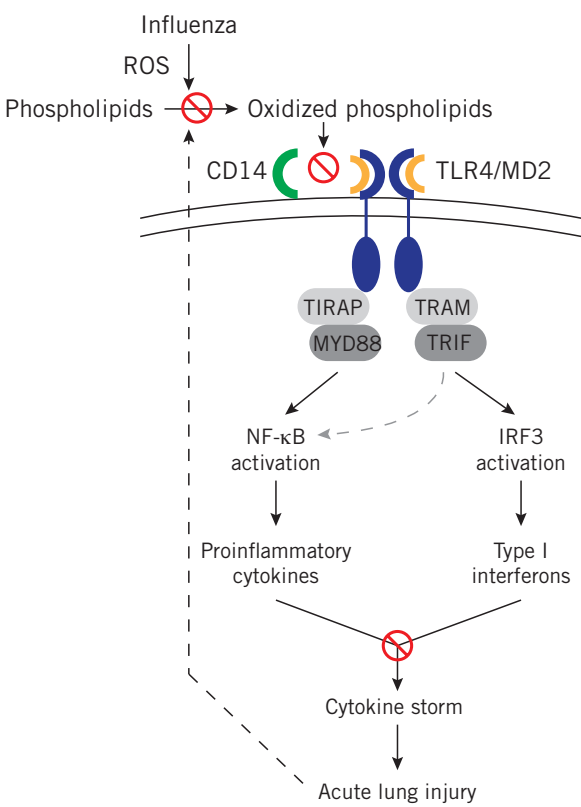


In Cd14-deficient mice, Eritoran did not provide protection to mice challenged with influenza. Moreover, in cell-based studies, Eritoran impeded lipid transfer from Cd14 to $\mathrm{Md}$ 2, suggesting Eritoran's protective effects are dependent on CD14.

The team also showed that mice infected with influenza and treated with Eritoran had substantially lower levels of oxidized phospholipids in their lungs than untreated mice, suggesting that Eritoran inhibits oxidized phospholipid production in response to infection.

Based on the results, Vogel proposed that Eritoran blocks oxidized phospholipidinduced TLR4 signaling, which mitigates the cytokine storm and blocks further production of phospholipid oxidation induced by pathogens (see Figure 1, "Proposed model of Eritoranmediated protection in influenza infection").

Results were published in Nature.

Dennis Voelker, professor of biochemistry, molecular genetics and medicine at National Jewish Health, thinks the strategy provides a practical approach for dealing with the inflammatory consequences of viral infection and could help improve the overall response of the host to a virus.

Voelker is developing the antiviral and anti-inflammatory palmitoyloleoyl-phosphatidylglycerol (POPG) as a prophylactic and therapeutic for respiratory syncytial virus (RSV), influenza A virus, acute respiratory distress and acute lung injury. ${ }^{5-7}$

"Importantly, the findings provide a new route for dealing with patient populations that fail to receive immunization or that respond poorly to immunization," Voelker said. "The approach also provides a new avenue for treating emerging influenza A strains that are resistant to neuraminidase inhibitors, which currently constitute the primary therapeutics for patients without adequate immunity to the virus."

Vogel added that "because Eritoran has a very good safety record in people, especially very sick people, translation into the clinic would be a reasonable possibility assuming that further preclinical studies support our initial studies carried out in mice and cotton rats."

\section{Collecting tolls}

The findings could point to a new opportunity for Eritoran and other TLR4 inhibitors, as well as for other compounds that failed in sepsis but work by stemming the cytokine storm.

“Treating sepsis is tough because you're trying to intervene at such a late stage when the cytokine storm is extremely strong," said Vogel. "With influenza, these drugs could help the immune system work to fight infection and blunt further initiation and potentiation of the cytokine storm."

TLR4 inhibitors in the clinic include Daiichi Sankyo Co. Ltd.'s CS4771, which is in Phase I trials to treat sepsis, and NovImmune S.A.'s NI-0101, a humanized $\mathrm{mAb}$ in Phase I testing to treat autoimmune disease and inflammation.

VBL Therapeutics Ltd. has VB-201, a TLR2 and TLR4 antagonist, in Phase II testing to treat inflammatory bowel disease (IBD) and psoriasis.
"We will continue to collaborate with Vogel's group on preclinical research," said Fabian Gusovsky, executive director of the chief innovation office group and special projects at Eisai Inc. and a coauthor on the Nature paper. "The mechanisms uncovered with our current work are certainly of interest, and as part of our human healthcare mission, we will continue to look at our compounds and evaluate opportunities."

Eisai declined to discuss any patent applications.

"We want to test Eritoran in combination with standard antivirals in influenza-infected mice and cotton rats and then extend our findings to ferrets. But we are not only limited to using Eritoran. We propose to test other agents that block TLR4 or its key signaling pathways," said Vogel.

Immediate plans for the Maryland team include testing the drug in combination with current antiviral agents, as well as in an aged cotton rat model of influenza infection from Sigmovir Biosystems to determine the potential of Eritoran in elderly individuals who respond poorly to influenza immunizations.

"Because the drug showed efficacy against multiple strains of influenza A, the approach is likely to have broad applicability and could be useful against other viruses such as respiratory syncytial virus, for which there is no vaccine," said Voelker.

Indeed, Vogel said, "we've seen some very interesting effects of Eritoran in animal models of bacterial infection and would like to be able to expand those studies as well."

According to Vogel, the University of Maryland, Baltimore has filed a new use patent for the compound.

Baas, T. SciBX 6(19); doi:10.1038/scibx.2013.453

Published online May 16, 2013

\section{REFERENCES}

1. Shirey, K.A. et al. Nature; published online May 1, 2013; doi:10.1038/nature12118

Contact: Stefanie N. Vogel, University of Maryland School of Medicine, Baltimore, Md.

e-mail: svogel@som.umaryland.edu

2. Imai, Y. et al. Cell 133, 235-249 (2008)

3. Nhu, Q.M. et al. Mucosal Immunol. 3, 29-39 (2010)

4. Opal, S.M. et al. JAMA 309, 1154-1162 (2013)

5. Numata, M. et al. Proc. Natl. Acad. Sci. USA 107, 320-325 (2010)

6. Baas, T. SciBX 3(2); doi:10.1038/scibx.2010.35

7. Numata, M. et al. Am. J. Respir. Cell Mol. Biol. 46, 479-487 (2012)

\section{COMPANIES AND INSTITUTIONS MENTIONED}

Cincinnati Children's Research Foundation, Cincinnati, Ohio Daiichi Sankyo Co. Ltd. (Tokyo:4568; Osaka:4568), Tokyo, Japan Eisai Co. Ltd. (Tokyo:4523; Osaka:4523), Tokyo, Japan

Iowa City VA Health Care System, lowa City, lowa

National Jewish Health, Denver, Colo.

Novlmmune S.A., Plan-les-Ouates, Switzerland

Sigmovir Biosystems Inc., Rockville, Md.

The University of lowa, lowa City, lowa

University of Maryland, Baltimore, Md.

University of Maryland School of Medicine, Baltimore, Md.

VBL Therapeutics Ltd., Or Yehuda, Israel 